\title{
The Design and Implementation of Gas and Liquid Flow Simulation System in Pipeline Network
}

\author{
WANG Peishuai ${ }^{1}$, ZHAO Gang ${ }^{2}$, CAO Xian ${ }^{3}$, WANG Wei $^{4 *}$ \\ Beihang University \\ Beijing, China
}

\begin{abstract}
This paper introduces some effective methods about gas and liquid flow visualization simulation in complex pipeline network with the purpose of decreasing the dependence on the topology of the pipeline network. A method was proposed to define the parametric information of the topology of pipeline network and the shape, size and position of each pipeline. The parameter driven modeling is achieved in simulation runtime and used to discretize the pipeline model into pieces in order to simulate the fluid flow process more vividly. A method based on graph theory and pipeline state was proposed to realize the gas and liquid flow simulation in the pipeline network of arbitrary topology. Finally a typical simulation system was designed and implemented to validate the above methods.
\end{abstract}

Keywords-pipeline network; parametric modeling; discretization; graph theory; visualization simulation

\section{INTRODUCTION}

The pipeline network system has been used widely to transport the gas and liquid in many fields such as aerospace, automobile, shipbuilding, water resources, hydropower and medical hygiene. Most of those pipeline network systems consist of many pipelines (such as straight pipeline or arc pipeline) and many stop valves that are used to control the flow routine of the gas and liquid.

The visualization simulation about the gas and liquid flow process in the pipeline networks has strong demand because the simulation could show intuitively the running process of the system and show the real time data, as well as verify feasibility of the project and so on ${ }^{[1,2]}$.

At the present stage most of the existing simulation systems rely heavily on the specific topology of pipeline network system. For example, in modeling stage, the model was created by some commercial software in strict accordance with the pipeline network topology before the simulation, and in simulation stage, designers will analyze manually the gas and liquid flow routine according to the stop valves states, and design laboriously the simulation process.

However the topology of the pipeline network system could need to be modified frequently for a variety of reasons, which will lead to redo almost all the works, like rebuilding the model, redesigning the flow routine and simulation process.

This paper is sponsored by the National Science and Technology Major Project (2014ZX04001-081-07) and the National Defense Project of 863 (2015AA1406).
In order to solve the problem of heavy dependence on the topology of pipeline network system for simulation system, the research of gas and liquid flow visualization simulation in complex pipeline system was carried out on the basis of previous work.

A method was proposed to define the parametric information about the topology of pipeline network system and the shape, size and position of each pipeline. The parameter-driven modeling and pipeline discretization method was proposed. A method based on the graph theory and pipeline state was proposed to realize the gas and liquid flow simulation in the pipeline network of arbitrary topology.

Finally, a typical simulation system was designed and implemented to validate the whole solution, and realized a gas and liquid flow visualization simulation system that adapted to arbitrary topological pipeline network system, which has a satisfactory result in practice.

\section{PARAmetric Definition Of PiPELINe Network System}

In order to decouple the pipeline network topology from the simulation system, a method was proposed to define the parametric information about the topology of pipeline network system and the shape, size and position of each pipeline.

The XML script was used to define the parametric information of the pipeline system by a specified rule. The parametric information includes the topological information, which is expressed by the connection relations between a pipeline and a stop valve for example:

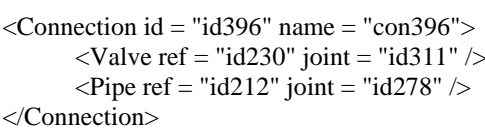

The topological information also includes the shape, size and position information of each pipeline, for example (a straight pipeline and an arc pipeline):

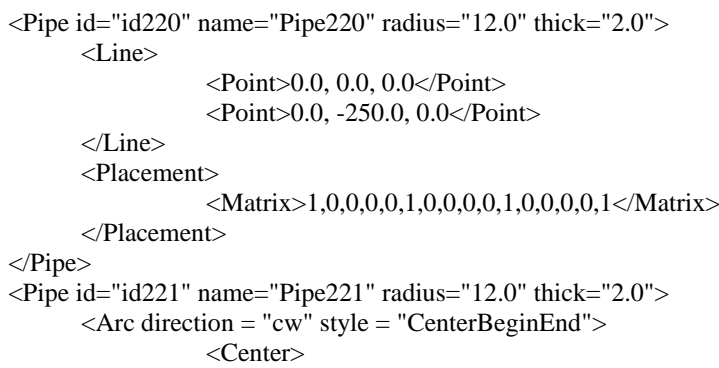




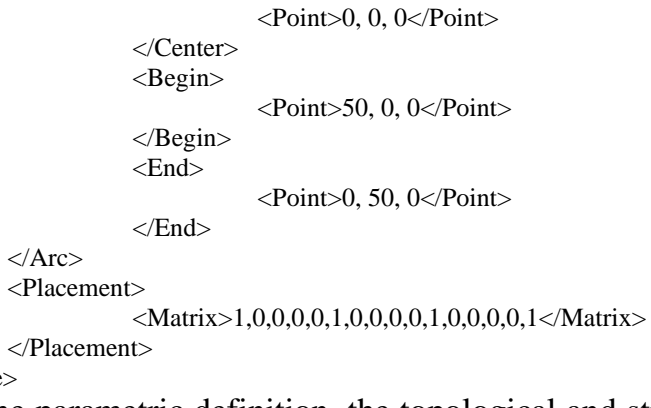

By the parametric definition, the topological and structural information was confined in the script and will influence straightly the simulation process. Once the topological and structural information is changed, we just need to modify the XML script and the simulation system will automatically adapt to the change.

\section{PARAMETER DRIVEN DISCRETIZATION MODELING}

On the Basis of the above parametric definition, a parameter-driven modeling method was proposed.

First, read and parse the parametric information in the simulation system. Then use some $\mathrm{C}++$ solid modeling toolkit to construct the pipeline models according to the shape, size and position of each pipeline. The pipeline models refer to the triangular patches. Finally render the models in the simulation scene.

Instead of generating the whole pipeline model, we discretize the pipeline model into many segments ${ }^{[3]}$ in order to simulate the liquid flow process as in Fig. 1.

For example, in order to simulate the process that liquid flows through a pipeline from one endpoint to the other endpoint, it will obtain satisfactory results to gradually change the color of each segment in the series segments of the whole pipeline model. The smaller segment we divide, the more vivid result we will get.

\section{The Simulation Method Based on Graph Theory AND PIPELINE STATE}

On the basis of the parametric definition of pipeline network system topology, a simulation method based on graph theory and pipeline state was proposed to realize that the program can deploy the simulation automatically according to the topological parametric information.

\section{A. Graph Theory Model}

First a graph theory model was abstracted from the pipeline network system which consists of many pipelines and many stop valves ${ }^{[4,5]}$.
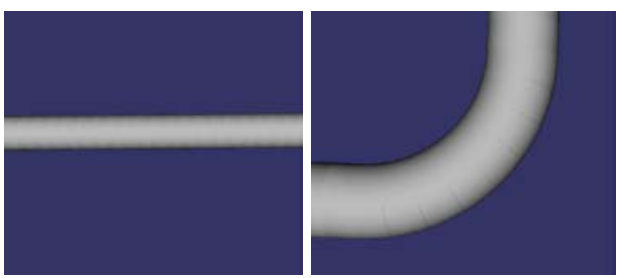

Fig. 1. parameter-driven modeling and discretization
The whole pipeline system was abstracted as a graph; define each stop valve in pipeline system as an edge; and define several pipelines as a node in graph. Those pipelines have a basic feature that they are connected straightly with each other and they are not divided by any stop valve into different parts. For example, a simple pipeline network system shown in Fig. 2 could abstract a graph shown in Fig. 3. Each stop valve in the pipeline system corresponds to an edge in the graph, namely $e_{i}$ represents $v_{i}$, and the mapping relation between pipelines in the pipeline system and nodes in graph are shown in TABLE I.

According to the graph model, an adjacency matrix as following was build:

$$
\mathbf{A}=\left[\begin{array}{cccccc}
0 & \mathrm{~S} 1 & 0 & 0 & 0 & 0 \\
\mathrm{~S} 1 & 0 & \mathrm{~S} 2 & 0 & \mathrm{~S} 3 & 0 \\
0 & \mathrm{~S} 2 & 0 & 0 & \mathrm{~S} 4 & 0 \\
0 & 0 & 0 & 0 & \mathrm{~S} 5 & 0 \\
0 & \mathrm{~S} 3 & \mathrm{~S} 4 & \mathrm{~S} 5 & 0 & \mathrm{~S} 6 \\
0 & 0 & 0 & 0 & \mathrm{~S} 6 & 0
\end{array}\right]
$$

In which we define:

$$
S_{i}=\left\{\begin{array}{l}
0: \text { when the stop valve vi was closed } \\
1: \text { when the stop valve vi was open }
\end{array}\right.
$$

Before the process of simulation, the graph theory model was abstracted first from the parametric definition information Then the adjacency matrix was generated. In the process of

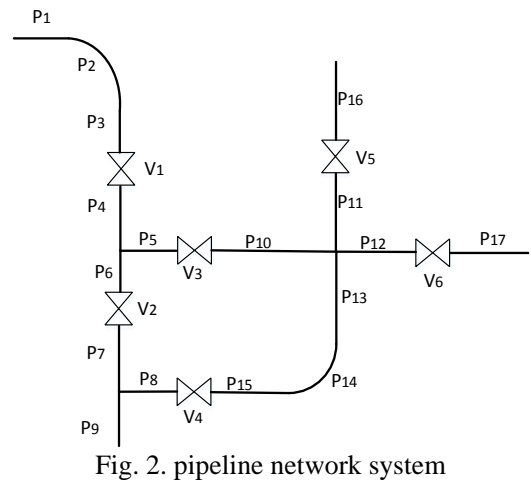

simulation, the adjacency matrix was updated in real-time

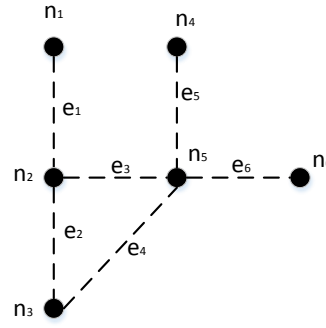

Fig. 3. graph model

TABLE I map relation between pipelines and nodes

\begin{tabular}{|c|c|}
\hline $\mathrm{n}_{1}$ & $\mathrm{p}_{1}, \mathrm{p}_{2}, \mathrm{p}_{3}$ \\
\hline $\mathrm{n}_{2}$ & $\mathrm{P}_{4}, \mathrm{p}_{5}, \mathrm{p}_{6}$ \\
\hline $\mathrm{n}_{3}$ & $\mathrm{P}_{7}, \mathrm{p}_{8}, \mathrm{p}_{9}$ \\
\hline $\mathrm{n}_{4}$ & $\mathrm{p}_{16}$ \\
\hline $\mathrm{n}_{5}$ & $\mathrm{p}_{10}, \mathrm{p}_{11}, \mathrm{p}_{12}, \mathrm{p}_{13}, \mathrm{p}_{14}, \mathrm{p}_{15}$ \\
\hline $\mathrm{n}_{6}$ & $\mathrm{p}_{16}$ \\
\hline
\end{tabular}


according to the state of each stop valve. And based on the real-time adjacency matrix, the program will simulate the gas and liquid flow process easily.

\section{B. Simulation Method Based on the Pipeline State}

A state was defined first for each pipeline in the pipeline system, as well as the state transition conditions. In the process of simulation, the pipeline simulation change only depends on its current state. For example, when a pipeline A is on "gas flow in" state, the gas pressure will get bigger in every frame in the render simulation process, and the pipeline color will become deeper, if the node containing pipeline $\mathrm{A}$ and the node containing pipeline B in the adjacency matrix is adjoined, the state of pipeline B will be set to "gas flow in" as pipeline A. When the gas pressure reaches max value, the states of both A and B will turned to "full of gas".

The state is divided into two kinds: stable state and unstable state. The pipeline on stable state does not have simulation change, while the pipeline on unstable state will change in simulation process. When all the pipelines are on stable state, this simulation process is balanced and finished. If any stop valve is turned on or turned off, the balance is broken and another simulation process begins.

The major feature of the complex pipeline network system is that when one pipeline state is changed, the adjacency pipeline that is intercommunicated with that pipeline may change its state also. It is easy to judge whether two adjacency pipelines are intercommunicated according to the adjacency matrix generated above.

\section{THE SYSTEM VERIFICATION}

The methods proposed above together make up the solution to decouple the topology of pipeline system from the gas and liquid flow simulation system, and decrease the dependency on topology. In order to verify the feasibility and result of the solution, a typical gas and liquid simulation system was designed and implemented.

\section{A. Simulation System Structure}

As in Fig. 4, there are four tanks A, B, C and D. The tank $A$ is full of high-pressure gas, and is used to provide motivation for the whole pipeline network system.

The four tanks are connected through a complex pipeline network system which consists of many pipelines and stop valves, and the pipelines are divided into straight pipelines and arc pipelines according to their shape. The end-point of each pipeline can be connected with another pipeline, a stop valve , a tank or the environment.

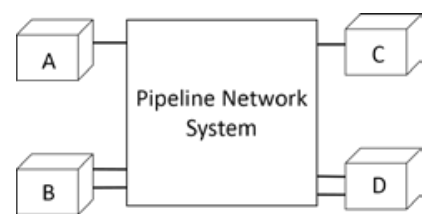

Fig. 4. structure of the typical simulation system
In this system, the four tanks and the interfaces between tanks and pipeline network system are explicit, while the pipeline network system internal topological structure and shape, size and position of each pipeline are flexible, and sometimes will change.

\section{B. Simulation Process}

The Fig. 5 is the topological structure of a typical pipeline system. A XML script is used to define the topological information and the pipeline shape, size and position information by the parametric way.

A C++ geometry modeling toolkit was used to model all pipelines, and discretize them into continous segments. The Fig. 6 is the generated model according to the topological structure shown in Fig. 5.

Then according to the topological information, the graph model was extracted and the adjacency matrix was generated and updated in real time.

The state graph of a pipeline is shown in Fig. 7. In the simulation process, each pipeline is visually simulated on the

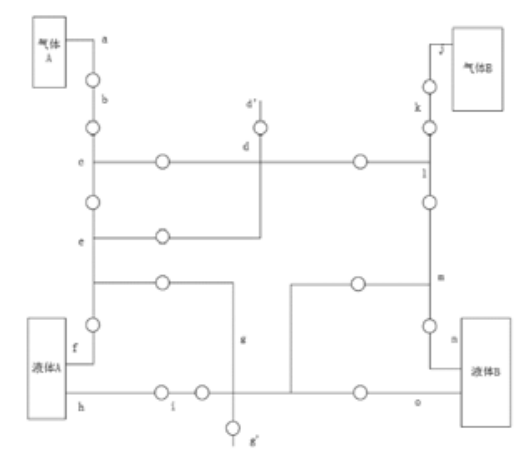

Fig. 5. topological structure of the typical simulation system

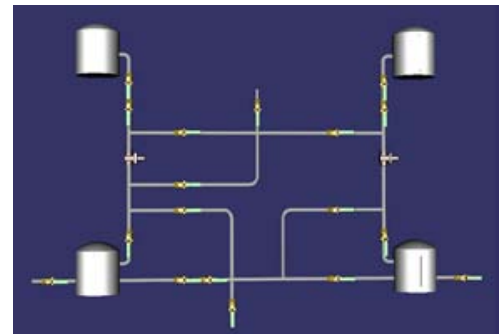

Fig. 6. the whole model generated by parametric information

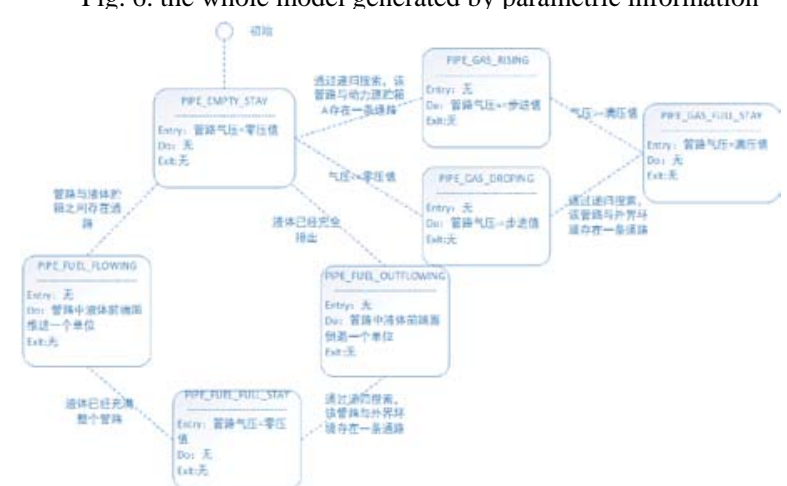

Fig. 7. state graph of a pipeline

rule of the state and the state transfer condition. 
The method is based on the state of each pipeline, making it possible to be independent of the concrete topological structure of pipeline system by simulation on its state rule in real time.

\section{Simulation Result}

The Fig. 8, Fig. 9 and Fig. 10 illustrate simulation effect for gas flow process, liquid flow process and the mixture flow process in the complex pipeline network system. The blue color represents gas while the red color represents liquid.

In the view of the simulation effect, the method proposed above is feasible and efficient, and improve the system flexiblility. It turns out that the solution will realize that the gas and liquid flow simulation system does not depend strongly on the topological structure of the complex pipeline network system.

\section{THE CONCLUSION}

The paper discusses how to discrease the dependence on the topological structure of pipeline network system in the gas and liquid flow visualization simulation system, and how to improve the flexibility and the adaptation of the simulation

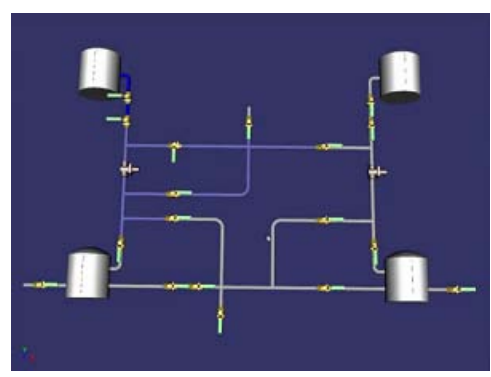

Fig. 8. gas flow process simulation effect

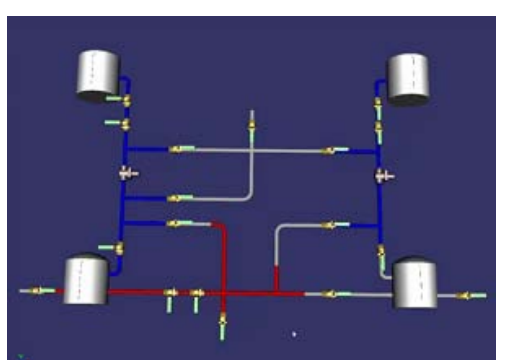

Fig. 9. liquid flow process simulation effect

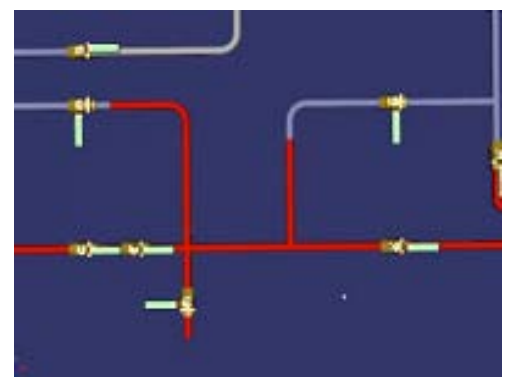

Fig. 10. gas and liquid mixed flow simulation effect system. Some practical and efficient methods are proposed, and a typical simulation was designed and implemented to validate the whole solution.

The parametric information definition about the topology of pipeline network system decouple the topological structure from the simulation system. The parameter-driven modeling depend only on the parametric information above, and the pipeline discretization method makes it easy to simulate the liquid flow process. The method based on graph theory and pipeline state realizes the gas and liquid flow simulation in the pipeline network of arbitrary topology.

About the gas and liquid flow simulation in complex pipeline network system, there is still a lot of work to do to improve the field:

It will be useful to monitor the pressure, flow rate and flow volumn of the gas or liquid in pipeline on line, and transport the data and show them in the simulation system for troubleshooting.

It will be useful to model simple fluid dynamics to simulate the fluid flow more vividly.

\section{Acknowledgment}

This paper is sponsored by the National Science and Technology Major Project (2014ZX04001-081-07) and the National Defense Project of 863 (2015AA1406). We sincerely thank the friends in National University of Defense Technology for their help.

\section{References}

[1] M. Nordsveen,A. Hærdig. "Simulations of severe slugging during depressurization of an oil/gas pipeline". Modeling, Identification and Control. 1997.

[2] Ortega-Mier,Garcia-Sanchez, M,Goti, A,A.. "Use a simulation tool for multi-commodity pipeline scheduling". Hydrocarbon Processing. 2013.

[3] A. Herrána, J.M. de la Cruzb, B. de Andrésb. “A mathematical model for planning transportation of multiple petroleum products in a multipipeline system”. Computers and Chemical Engineering. 401-413. 2010.

[4] Masashi Shimada1. "Graph-theoretical model for slow transient analysis of pipe networks”. Hydraulic Engineering. 115:1165-1183, 1998.

[5] Rajiv Gupta1, T. D. Prasad2. "Extended use of linear graph theory for analysis of pipe networks”. Hydraulic Engineering. 126:56-62. 2000. 